\title{
When Google Wave Hits the Pediatricians!
}

Google introduced Google Wave (http:// wave.google.com/) in June 2009, and is now inviting interested people to log on and check the tool.

\section{What is Google Wave?}

According to Google, "Google Wave is an online tool for real-time communication and collaboration. A wave can be both a conversation and a document where people can discuss and work together using richly formatted text, photos, videos, maps, and more. In Google Wave you create a wave and add people to it. Everyone on your wave can use richly formatted text, photos, gadgets, and even feeds from other sources on the web. They can insert a reply or edit the wave directly. It's concurrent rich-text editing, where you see on your screen nearly instantly what your fellow collaborators are typing in your wave."

\section{How Shall It Help Pediatricians?}

Improving patient-doctor interaction: As the waves can be easily embedded into any website, Google wave can be a potential application for central communication and collaboration tool for telemedicine practices. Now, the pediatrician can be connected to the patient in real time, check the reports online, put comments on the reports and respond. This whole conversation and the embedded document can be saved on the hospital database.

Intergroup interaction: The various specialty groups can have their own wave and discuss the common issues via online presentations, online conferences and podcasts, which all colleagues across the globe can attend at the same time. A pediatric nephrology wave has been created, you can e mail at sidsdoc@gmail.com to participate.

Drafting and publishing a manuscript: The Google Wave has everything we ever dreamt of for writing a manuscript. The author comes online, starts writing the document, invites other authors, and gets the inputs straight into the document. The charts and graphs can be easily made and linked to spreadsheets and other programs.

Using Igor for inserting references into the manuscript: Using Igor (http://code.google.com/p/ helpmeigor/), the user can simply drag in the references from Pubmed or from personal libraries like CiteUlike, into the article one is writing.

Google Wave can also be an important tool in implementing the medical home concept endorsed by the American Academy of Pediatrics (AAP). The medical home concept fosters an ongoing relationship with a personal physician trained to provide first contact, continuous and comprehensive care through all stages of the patient's life, including during acute care and chronic care conditions. Physicians will require using the new web 2.0 tools such as Google Wave to efficiently provide this type of care.

Sidharth Kumar Sethi sidsdoc@gmail.com 